rest with respect to the nature of their malady, when it was fully explained to them that the experiment would be attended with little or no pain, and that the commencing chancre artificially produced could with certainty be destroyed on or before the fifth day, and probably later, without the slightest chance of the constitution suffering from what had been done, while his medical adviser would have obtained important information for conducting the treatment. After the use of the caustic, a piece of dry lint, rather larger than the white eschar produced, should be applied, in order to protect the surrounding or opposed parts; and this is all that will be required when the sore is on the inner prepuce, or on that part of the glans which is covered by it; butin exposed situations the lint would become adherent to the edge of the ulcer, and cause pain and inconvenience on its removal, unless covered by some dressing which would prevent evaporation. A larger piece of lint soaked in a mild astringent lotion, and covered with a strip of oiled silk, or simply a piece of lint, upon which some mild uniritating ointment has been spread, will answer this purpose. Should any inflammation be present, or be anticipated, rest may be enjoined with the horizontal position, and the surrounding parts kept wet with Goulard lotion, or simple water, either in the form of poultice with bread, or by means of linen or lint dipped into them and laid over the parts. When the alcer is seated on the prepuce, these local precautions are especially advisable, as a slight amount of inflammation is in this situation soon followed by serous effusion into the cellular tissue between the two layers of this membrane, and phymosis or paraphymosis would be quickly indaced. The penis under these circumstauces should be surrounded by the lotion, and supported against the abdomen. Low diet and saline aperients may also be required, or a more severe antiphlogistic treatment may be called for.

\section{HEPATIC DROPSY.}

By R. DAY, Esq., Surgeon, Wymondham, Leicestershire.

This disease is known by the large abdomen fluctuating on percussion; now and then anasarca in the legs, from pressure of veins above; pain or tenderness in the region of the liver; often tip of right shoulder, or between the scapulæ; the patient sometimes lying on right side or back, aud now and then experiencing a dragoing sensation when attempting to lie on the opposite one; nu icterode appearance of countenance or eye at one time or other; clay-coloured stools; dwindled face and extremities.

I have seen elaterium sink the patient in a short time. Six-drachm or one-ounce doses of cream of tartar I have known distress much; diuretics have often failed. Tonics I should prefer, since the structure of the liver is too far disorganised in the more adranced cases (the recurrence and extent of the ascites being the best index of the amount of disease the organ has sustained), and the constitution too much impaired by previous intemperance of living, to expect advantage from any but the most mild means. Mercury could not but do harm, particularly when ptyalism is rapidly induced, as all medicines contributing to debilitate. Some benefit, I think, might be derived from the use of purgative enemas. I have seen the following remedy of avail in two or three cases :-

R Hydriodate or iodide of potass, $Э \mathrm{iv}$; Nitric ather, $\mathrm{f} \xi \mathrm{j}$;

Infision of cascarilla, $\mathrm{f} \xi \mathrm{xv}$;

Compound spirit of lavender, f 3 iij. M. A wineglassful twice a-day. Meat diet; no beer or spirits : for it would appear that these latter exert some specific influence upon Glisson's capsule, ramifying throughout the structure of the liver, and so tightly contracting the vascular tissue, as to prevent the circulation of the portal blood, thereby distending the abdominal venous system, and creating the ascites. Every medical man must have noticed the distress from flatulency and indigestion, \&c., which purgatives by the mouth must exasperate.

As to the time that paracentesis abdominis should be performed for the removal of the ascites, I see no valid grounds to dissent from the principles applicable to each variety, adranced at the close of the paper on cardiac disease. Acupuncturation might possibly cast into the shade the operation of puncture by trocar ; but this latter might be more available if earlier performed: for the surrounding parts would then be more healthy, and the patient's constitution better capacitated than subsequently. In two or three cases of long-protracted ascites, the sufferers have died a few days after the operation with symptoms of chronic peritonitis; and on inspection post-mortem, a dark Modena-purple state of peritoneal membrane and adjacent tissues has been manifested.

When we consider the cause of the ascites, we need not wonder at the failure of remedies, and therefore should not trust too long or too exclusively to their use, but proceed at once to a more obvious source of relief. If we could get rid of the dropsy by the simple means above specified, some time would elapse before the fluit would again collect : to this resource we might, if necessary, recur, and save the patient the extra. trouble, and often danger, of taking medicine, and then only of an inoffensive kind.

The recollection that the organ is diseased (and the same observation is applicable to any organic disease), and often far disorganised, and infinitely beyond the powers 
of amendment by any medicines, as experi-|been credibly informed about that of the ence has proved, is too much lost sight of: healthy organ.

in the case of the disease under our notice, if but a third part of the liver were healthy, what a relief should we not have to the circulation-debarred of this we nust endeavour to compensate by unloading the stomach of indigestible substances. I see no objection to a gentle emetic occasionally, as ipecacuanha, Эj, tartar-emetic, gr. $\mathrm{i}$; and a slight aperient now and then, which would arail to prevent those sicknesses and diarrhoeas and headachs that are sure to ensue at almost stated intervals, and which may be anticipated by errors of diet and changes in the conditions of the atmosphere.

A trial of alteratives, as Plummer's pill, with tonics; the new preparations of iron (as many of them are said to be not disagreeable), or some of the salts of iodine, or even minute doses of antimony scarcely sufficient to nauseate, might avail, since there must be something sub-inflammatory going on to account for the thickening and contraction of the organ perceptible by the eye throughout its structure; the system being at the same time supported by a thoroughly nutritious diet, i. e., with a good allowance of animal food, permitting, if necessary, a couple of ounces of sherry wine a-day-debarring the use of fermenting and stronger vinous liquors.

When I first entered the profession, having the good fortune to commence my studies in an hospital, my attention was particularly attracted by patients (especially in the milkdiet class) complaining of their milk disagreeing with them; some said it curdled in their stomach, and was rejected in this state; some that they could not keep it doun; others that it created the most uneasy sensations possible, which sometimes lasted for hours ; and one here and there that it caused diarrhoea. To all these complaints, ever and anon made by wholesale by the patients, the medical officers turned a deaf ear. Perhaps this superlative obstinacy of rejecting testimony on the large scale will now give way under the sound and enlightening views of Dr. Prout. This consummate physician has satisfactorily shown that milk is by no means an easily digestible fluid, and that some powers of stomach are necessary to convert its component (viz., oleaginous, albuminous, and saccharine) principles, into a pabulum that may admit of easy assimilation.

For the subduction of the local inflammatory action, cupping, cataplasms, blisters, or mercurial plasters, might be useful. In two or three cases I have seen the liver so contracted as to give its surface a strictly honeycombed character, and studded over with fossulæ, each capable of holding a tolerably-sized pea, and the general bulk of the organ half its natural size and tucked under the diaphragm. The weight I have not ascertained, but I have

Cardiac dropsy remains to be considered. As I have paid some considerable attention to this subject, I shall endeavour so to mould the arrangement of it as will enable any one, to whom opportunity has been wanting to mature his experience, to obtain a short and ready way to a tolerably correct diagnosis. I must premise the use of the stethoscope, and some little acquaintance with the natural sounds of heart and lungs. As we meet with dropsy from diseased heart in all its forms, a minute description would not only be prolix, but rather confuse, and therefore I shall give the broad outlines, and recommend a most useful little work on dropsy by Dr. Seymour, from the perusal of which I first obtained considerable aid in diagnosis of the dropsies, of which I have been treating.

First. If a patient inform me that he has had dropsy over the greater portion of the body, and that his breathing is affected on slight exertion, my suspicions are instantly raised to the heart as the cause.

[To prevent vitiation by renal disease, a common coincidence, the urine should be unalbuminous and the face ruddy.]

Second. If the patient has had rheumatism, the case scarcely admits of a doubt; still less if there have been with it palpitation or pain in the pericardiac region.

[The rheumatism is generally of a character to create pain, heat, and swelling about joints or muscles; but such an acute attack is not necessary, since $I$ have found the heart diseased with a loud, blowing sound, where there has been very little, if any, rheumatism in two or three instances.]

Third. The patient will now and then have had no dropsy for months, but more dyspnoa than natural. Suspect the heart, particularly if there be sanguineous complexion.

[In two or three cases of this kind, I have found a blowing sound two or three inches around heart's apex, and sometimes loud enough to be distinctly heard with the pa. tient's dress on.]

Fourth. Now and then a peculiar jerking, vibrating, slightly bounding pulse, closely approximating to the hæmorrhagic, will alone point out the heart as diseased: Dr. Hope's pulse of aortic regurgitation, of whom I was taught its nature. In one instance I was able to make out an aneurism of the aorta from this state of pulse, arresting my attention to the heart; there was no dropsy; a loud murmur in the course of aorta; strong impetus opposite the second costal cartilage, considerably louder than at heart's apex; absence of natural resonance for a couple of inches or more; pain in the part increased in intensity, from ten, p.m., to two, a.m., extending to fingers of left hand; wandering pains in chest. He was a slender, little. 
man. He was relieved by morphia; went out of hospital with the privilege to return when he should become worse; retat. 36, or thereabouts; a shoemaker by trade. I forget whether he ever had rheumatism since. This pulse I have never found unconnected with rheumatic pericarditis, or endo-carditis; in two cases of aneurism traced to the cleadrooms, its absence I noted in both. The pulse with the thrill I have remarked six or eight times.

Fifth. Any one acquainted with auscultation will easily discover, by the application of the naked ear or stethoscope to the chest, any morbid murmur or blowing sound synchronous with the pulse, and differing from the respiration by the number of whiffs being from twenty to thirty in a minute; whilst the cardiac soruds may be eighty, ninety, or upward: in an equal time. By the same means ve obtain every information in respect to the heari's impulse-its regularity; as, also, the general state of the pulmonary organs.

Sixth. If there have been previous or abiding anasarca, with a strong engine-like action of the heart, this organ is at fault : there may be present with this the blowingsound or not, or a heaving impulse of the ventricle, distinctly raising the head and stethoscope from the patient's chest. Any or all of these are diseased conditions.

[Sometimes the murmur is imperceptible or slight, the heart's contraction smart, and a doubt may arise as to the precise state of the organ. If there be no anæmia, or previous loss of blood, and a rheumatic attack can be traced; if leaping on a bench three or four times induce a luuder bruit or whiff, and stronger impulse (in anæmia, \&c., the impulse wants pouer), we may safely conclude the heart to be damaged.]

Seventh. If the chest be distinctly raised at each inspiration, and the respiration extremely puerile, and sonorous rhonchi, or musical notes, transmitted; oftentimes, in consequence of the engorged state of the lungs, they can be felt rubbing against the parietes of the thorax, so as to constitute the friction ascendant and descendant; the lips will be livid and the dyspnoea severe. Though the heart will generally be affected, yet I have often known such cases to be connected with kidney disease.

Eighth. The expectoration furnishes use. ful corroborative testimony. In congestion of the langs, from obstruction to heart's circulation, the sputa are thin and mucous, wanting the whiteness and frothiness of bronchitis, and the viscidity of pneumonia, and brownish, from admixture. with the colouring matter of the blood.

Ninth. The position of the sufferer must. not be disregarded. The patient is obliged to have himself raised and supported by pillows in the sitting posture; there is orthopnoa, and the patient's breathing would be intolerable were he to assume the recumbent position. He will retain this attitude day and night for weeks.

[Nuch the same portraiture is presented by patients suffering from extensive renal anasarca, as also hepatic : a little care will prevent error of diagnosis.]

Tenth. Where the dropsy pervades the upper extremities, thoracic and abdominal parietes, and integuments of the loins, we may connect the fault to the heart.

As a great general rule, where persons make a great fuss about their heart beating violently, and other symptoms of nervousness or nervelessness appear, we are assured of its functional derangement only, and should remove the ill-forebodings of the patient.

Generic Distinctions. - These are two: the centric or active hypertrophy; i. e., increased muscular parietes of the ventricle, right or left, as the case may be, the cavity remaining the same, or slightly diminished; and the eccentric or passively dilated heart, with (?) or without addition, perhaps diminution of muscular structure; the ventricular cavity, right or left, according as the tricuspid or mitral valves are diseased, being increased.

The first species occurs generally in robust and large-framed persons, who have inured themselves to copious draughts of ale and porter, and strong exercise, lifting heavy weights, \&c., such as our coalheavers, porters, \&c. These characters will often consume ten or twelve pints a-day, and some double that quantity; they are extremely muscular, possess huge bloated faces, are full of blood, such as it is. They often observe at first some puffiness about the eye, and more or less lividity about the lips; then odema of the ankles and dyspnoea. Should this go to any greater extent, these individuals merely fancy they have taken cold, and failing to obtain relief at the expected period, they seek an asylum in the wards of a hospital. Their case is clear: soon the anasarca extends up the legs and thighs, distends the scrotum and abdominal cavity-the latter to a less extent; the breathing is short and gasping; chest sometimes unnaturally resonant, and pervaded by sonorous rhonchi, sometimes duller than natural; and then the lips and face are more livid and the orthopnoea more urgent; the countenance pourtrays considerable suffering; the action of the heart may be tumultuous, irregular, contracting in rapid succession two or three times, and then appearing as though it turned over, and repeating this ceaseless round for days, the patient often expressing great pain in the cardiac region. The urine is generally scanty, bowels pelhaps torpid ; the swelling of the lower extremities increases, gradually infiltrating the cellular texture, so that it becomes difficult to pit them, and produce the stamp of the finger, and such a state is often designated by the term as "s hard as a board." In two 
or three weeks the legs give way, a considerable quantity of serum oozes therefrom, an unhealthy slough forms, and the patient dies a day or two afterwards.

Other cases present themselves in which the patient has been more observant of his living, has not perhaps engaged in such laborious services, and seeks relief on the first appearance of ailment; his anasarca pits, and is perhaps confined to legs and ankles. There is little or no ascites, and slight dyspnoea : perhaps a bleeding and two or three brisk purges restore him to apparent health; the action of the heart still continuing strong, raising the instrument, as may be demonstrated by placing it on the cardiac region, and putting the hand on its opposite end. Such a patient, by moderate care of himself, may continue free from anasarca for eight or eighteen months, or more, and fancy himself cured.

The second form of the disease, viz., the dilatable variety, occurs chiefly in women, between thirty and forty years of age, in whom the fibre is more lax. Often these persons have been stout and fat, as they express it, and after the lapse of a few years have become considerably thinner. It is said that washerwomen and gin-drinkers are more prone to it, as, indeed, those too whose services are more laborious. The opposite sex is not exempt; and sometimes a discussion will arise as to the dilatation being mixed with hypertrophy. Women sulfer less than men, perhaps in pursuance of their greater abstemiousness : the anasarca does not go to the same extent, and retains for a longer time the impress of the fingers. There is more difficulty in positively deciding in the form we are now entering upon, whether the central organ of the circulation is affected, especially where we have symptoms of hepatic disease; since the ascites goes to the extent of impeding the respiratory functions, and in some measure the free play of the heart is interfered with; and the latter, to overcome the obstruction to its circulation, is aroused to greater activity. There is the same anasarca, the result of pressure of ascitic fluid, upon the iliac veins.

The sounds of the heart are louder, and evidently wanting the engine power of the ventricle in its hypertrophied state. They extend over a greater space, and are attended with a flapping noise; there is no heaving impulse, the heart conveying the sensation of a succession of feeble jerks over that space which is the immediate seat of disease; the pulse is moderate upon the whole, sometimes a little fuller than natural. In brief description, a state is induced not much unlike to what happens where too much blood has been lost; the anasarca is not nearly so tense as in the preceding class; the face and upper limbs sometimes puffy, and sometimes not so,
Mere disease of the heart, active or passive, with valvular disease or not, may or may not of itself induce anasarca, since the latter may be absent for months, and longer; two years' interval I have known. The history of some cases would seem to show that active disease had existed many years prior to any manifestation of dropsical effusions; and the irregular time at which they occur in many patients, and some even then in good apparent health, attest its dependence upon some unknown derangement of the system, perhaps of the capillaries.

It might have been expected that in hypertrophy we should have had a strong, hard pulse, or one possessed of pretty uniform character: the hypothesis does not accord in anywise with the results of my own investigation of cases.

The hurdest and most incompressible pulse I ever experienced'in eight years of hospital practice, occurred in two patients in the house at the same time, and singularly, side by side of each other; one of them died, and the other went out. The left ventricle would a little more than contain my thumb (whatever contraction might have gone on after death or before, I know not). There was no dropsy; the heart regular in its action; no impulse. The patient was a strong mason by trade. Fie died after having been in the house three or four weeks, suffering the last three or four days of his life all the symptoms of an aneurism compressing the trachea: he was not emaciated, but complained of debility.

With respect to the relative frequency of these dropsies, I should say in men cardiac is the most common; next renal; and, lastly, hepatic. In women, cardiac and hepatic nearly equal; renal more seldom. Had I intended to publish my own observations some time since, I would have kept a sta. tistical account. The quantity of urine in cardiac disease is scanty, and also in hepatic ; considerably more copious when the former is implicated with the kidneys.

In the generality of cases the pulse presents nothing particularly worthy of notice. A prolonged pulse is worthy of regard; sometimes it is unusually small and weak; it may be irregular, unequal, fluttering, and intermittent ; any of which states would engage attention to the central organ. The patient's appetite is often far from bad; not so inconstant as in many other diseases; there is less of flatulency than either in functional diseases of the heart, or in other organic diseases; neither is there the emaciation as in the latter.

One circunistance to which I have not yet alluded, is the alternately swelling and subsiding of the jugular veins, proving obstruction of the heart's circulation. Here, the heart has generally been very quick and irregular in its action; the column of venous blood instead of passing to the heart has re- 
trograded, occupying at first a third of the length, then two-thirds, and lastly the remaining portion of the vessel, as shown by its fulness and turgidity; and then the calibre of the vessel has completely collapsed, the contents, without further impediment, taking their natural circuit. This state warns us of the progress the disease has made; the countenance being livid, the dyspnoea urgent, and death soon closes the scene. The practised eye may observe a lesser degree of this retrograde course of the blood in the superficial veins of the neck in some persons, and to this extent it may not be the immediate precursor of death, for I hase known such patients live weeks.

I fear our curative measures will not avail much in this disease, since we cannot rectify the state of the organs upon whose soundness health so much depends. Since, however, the interruption to the circulation must tell somewhere, in what part are we so likely to perceive it as the capillaries, whose functions, especially in the lungs, from their proximity to the heart, most suffer; while their pervading every texture of the body will also explain the anasarca, the sure result of impediment to the onward progress of the circulating fluid. I shall preface this subject by a few brief observations. The capillary vessels, whose anatomy is more an induction of the reason than of the scalpel, and whose microscopic size bespeaks the cause of that, are the extremely minute ramifications that connect the peripheries of the arteries and veins, and which so thoroughly pervade every atomic portion of the body, that insinuate the finest needle where you will beneath the skin, there issue on its exit their crimson-coloured contents.

Without entering into the researches of microscopical observers (for even the microscope seems to give it different versions), we may take it for granted that their functions, no less than their physical properties, sufier considerably; and from what we see of the effect of blushing and pallor in the cheeks from varied emotions of the mind, we cannot but conclude their importauce to be such as is inferior to none in the whole animal system. We all know the relief of a congested lung by abstracting blood; the healthful effect of stimulating by ammonia and steel the system of a chlorotic female, and restoring the bloom to her lips and cheeks; and, Jastly, the great benefit to those suffering fron extreme debility and passive congestion in the lungs and legs (a modification of anasarca) from the use of the same remedies.

If we carry these principles in view we shall pave the way, methinks, to a more rational view of treating cardiac dropsy; for the practice now pursued fails too much, and the cause, $I$ believe, is attributable to the too much regard which has been paid to the names of diseases and to the pathology, not rightly followed out, and certainly far, very far less to the therapentic powers of remedies, of which the wonder to me is we know so much.

If we saw a man (a blacksmith) whose biceps humeri muscle was much hypertrophied, who would think of taking it away by antiphlogistic regimen? Should we not enfeeble his body at the risk of leaving his biceps as we found it! Is not the heart in the same predicament? The hypertrophy in the former instance was the over-nutrition, the providential, kind nature! result of the over-work imposed upon that one arm; has not the heart to overcome an obstacle, sometimes by an unallowable adhesion to the pericardium, sometimes by a rigid valve, and sometimes (we, human physicians, by not regarding nature's rules) by our weakening plan unknowingly, and truly unintentionally, call upon the heart to exert herself afresh to remedy our defects?

The well-known fact of over-distention of the veins by injections of water producing dropsy, might tend to illustrate the advantage accruing to bleeding, evacuants, \&c.; and experience has taught the absolute necessity of unloading the capillaries by the lancet, as also the instant relief to the patient by this temporary expedient. But the capillaries require some counter-check to the continued gravity of the blood, which their contractile powers are insufficient to overcome: hence the semi-erect posture assumed by the patient to free the heart of all the incumbent load of the lungs, whose inferior lobes are infiltrated throughout, and an opportunity, moreover, afforded for better oxygenating the vitiated carbonised air with which they are surcharged, and life threatened. It is doubtless on this principle of stimulating the capillaries that benefit flows fiom æther draughts, cordials, \&c., or the employment of a blister, or calefacient plaster, or dry-cupping, first to one part and then to another. If thus by removing congestions, where they exist, by a small bleeding, and preventing their recurrence, in some degree, by strengthening the system by nutritious diet (animal, the most natural), assisted by light bitters, abandoning everything that could stimulate the heart and endanger the capillary functions, we might guard it from that efflux of blood, and that inequality in the balance of the circulation, which irritability is sure to engender. Thus much for these most important vessels.

A question which has long agitated my own mind, and from the close connection it may have with the preceding topic, may not be here displaced, is, what is the condition that will most readily account for those sudden deaths of individuals in apparently better health, of which friends and bystanders are tolerable judges, and towards manifest (as we think) improvement in the eye of the practitioner. I have known, twice 
or thrice, dropsy disappear very gradually; the patient express satisfaction at his improvement for ten days or more; he has ate his meat, drank his porter, slept well, and is anxious to leave his bed. 'To this his physician has assented. That same day he Las suddenly died. I recollect another, on the strength of her improvement, quitting the hospital, and after a lapse of fourteen days or more as suddenly expirc. The most ready way to account for the suddenness of dissolution appears to accuse the heart's cessation, either from the too long-continued irritability of its muscular fibres, or the undue supply of blood to the brain, and a want of transmission of nervous stimulus to the circulatory moving force. The first explanation, I fear, we have no means of arriving at; and to the other there attaches considerable conjecture, by reason of the contractile power of the arteries going on some time after the last ventricular systole. In whatever view we regard the cause of death, it is too instantaneous to admit of the application of anything which might retard its progress, and afford time either for stimuli to the heart itself, by the external means of electricity, cold dash, \&c., to the thorax or medulla spinalis, or by the internal forms of spirituous and other stimulants. That time might permit of being treasured if the stress of the blood were imposed upon the capillary vessels, since we should have the forewarning symptoms of such an approach, and might apply the remedies accordingly.

The derangements of health apparently necessary to a speedy induction of auasarca, and which will only account for its supervention in those cases where there has been known to be, long since, a discase capable of its production at any period, must induce us to seek a remedy from those classes of medicines which seem specifically suited to the derangement under view. Our ingenuity is often taxed as to the precise derangement of function whence the dropsy immediately proceeds; and the patient's aspect, state, and description failing to inform us, our practice cannot but be empirical. There is no objection to the discriminate employment of diuretics in combination with tonics, and if the case last long the whole materia medica will bear to be ransacked. Rough remedies should be abandoned. Calomel, though I have had no experience of it in these cases, I should condemn. We are all acquainted with the febrile state of system induced by it after a brief continuance, and the irritable condition of the pulse, and of the languor the patient long complains of after a mercurial course.

In one case the sufferer had a most serious valvular disease; there was also an extremely loud murmur. The mitral valve was in such an ossific state, that it resembled more the introduction from without of a piece of rock-stone than anything of an in- ternal preternatural formation: he had also a diseased liver. He had been in this state for some time. Mercury was prescribed, which rapidly affected his system, and he died three or four days subsequently.

Colchicum has been condemned by those who have tried it, as might have been expected, from its irregular action. Elaterium I could have wished had shared the same fate. Some physicians are fond of it: this might be useful at the onset of the disease, where the powers of the patient are vigolous, but considering the obstacle to the circulation is an immovable one, and the patient's liability to be worn out by the obstinacy of the disease, and often the causes which have contracted it, and the repeated dropsies and other attendant evils, one cannot but be convinced of its ineligibility under these circumstances; and though I have seen no one carried off during the immediate operation of the drug, I am sure they have in several instances rapidly declined, and died a day or two after its employment.

Where the heart is very irregular, the pulse small, thready, and intermittent, the patient's breathing much oppressed, with intense orthopnoea, livid lips, and extensive anasarca, speedy relief may be obtained by digitalis, either in infusion or tincture, in union with other diuretics (two or three fluid drachms every four hours of infusion, or twelve to fifteen drops of tincture). This recipe, which I have seen Dr. Seymour enjoin several different times, and before now twice, if not thrice, in the same patient, has rendered the pulsexslower, fuller, and more steady, the breathing easier, and after a few days diminished the whole (or nearly so) of the anasarca, increasing considerably the excretion of the urine.

To oscupy further room in the discussion of remedial agents for a disease definite in itself, but protean enough in its forms, to which Iuxuries, on the one hand, and poverty on the other, besides other predispositions to disease innate in every constitution, have contributed, were a useless occupancy of your own valuable columns and the time of your readers, and a needlessly-imposed task upon myself; but ere I conclude, I must give a short sketch of the morbid anatomy of the affected organ.

Sometimes the pericaldium will be found universally adherent to the heart, and very much thickened by deposition of several layers of organised lymph. In one case there was a tendinous ball, probably a congenital malformation, tying the apex of heart to pericardium and central tendon of diaphragm. This case put on the aspect of dilated heart; the ventricle was by no means much hypertrophied; the apex seemed to me to strike the walls of the chest, making allowance for its being overlapped by the engorged lung. This patient was a shoemaker by trade, and seemed to suffer uneasi- 
ness more referrible to the stomach, as is usual in this class of operatives, from pres. sure of the last.

The size of the heart has been considerably larger than the patient's fist, the usual standard of admeasurement, and in some considerably more than double; the parietes of the left ventricle $a n$ inch or an inch and a fourth by guess; and I have seen the apex with its cavity formed solely of its own structure, the latter being surrounded by threefifths of an inch in breadth (perhaps more) of muscular tissue, totally obliterating the vertical interventricular groove.

The dilatable heart has occupied as much space as the hypertrophied form, particularly when a clot of blood has been enclosed in its cavity, and $I$ have seen it of sufficient dimensions to contain five or seven ounces of fluid. In such cases the organ has been very flabby in its texture, and the columna carnere loose, if I may employ the term. The valves I have found fringed, indurated, nodulated at their apices, one or more coated with a layer or ring of calcareous matter. I have seen this calcareous matter of such irregularity, hardness, and density, that if I had not been present on the removal from its position, I should have supposed some kind friend were practising on ny credulity, so much did it bear resemblance to a piece of rock that had been clumsily thrust into the substance of the heart. Now and then the heart has been rather freely encased in fat. In two instances I have noticed pulmonary apoplexy, the blood being so consolidated that one might have taken it for malignant disease. In these cases the tissue of the liver was here and there similarly infiltrated, and the venous system generally engorged. The pulmonary organs are variously congested. The brain $I$ have scarcely seen examined in such cases.

To terminate my remarks with elaterium, I am reminded fil'st of the topical means to be employed for "getting rid of the dropsy. Where all on usual remedial resources fail, I should say acupuncturation aflords the best possible chance, since it may be done at any time and place when the sufferings of the patient require. Indeed, I should notwait for these; but if there were much swelling $\mathbf{I}$ should direct it then, for the pressure must be injurious to the vessels and parts below, and to others at a distarice, as for instance the lungs, \&c. The making incisions is to be condemned, particularly where the auasarca has been of some standing and extent; for the structures have so far suffered thereby, that gangrenous sloughs have resulted, and the patient has died in forty-eight hours: of which unfortunate termination I could call to mind half a dozen examples. In early cases in which medicines failed at the onset, an incision might not augur such ill conse- quence; but why incur the risk even then, when the prick of a grooved needle may an. swer the purpose just as well?

Four or five different times $I$ have gone into the dead-room to visit the post-mortems; if the subject has been dropsical, and the integuments of the body throughout of a livid, gangrenous hue, as though some narcoticoacrid poison had been taken, $I$ instantly suspect the exhibition of elaterium to within a day or two of the patient's dissolution. In every such instance my surmises have proved correct. A patient dying of another disease, and bearing much the appearance the bodies above stated presented, induces me to quote the case.

A notorious drunkard having tumbled over a wheelbarrow, and shook himself a good deal, had himself bled; symptoms of delirium tremens coming on that night, he struggled much with his arms, amd lost a few more ounces of blood. He was brought to the house next day, and stimulants given : he died in the night. In thirteen hours, or seventeen the latest, his body was undergoing rapid putrefaction, his brain quite putrid, and chloride of lime was obliged to be used to destroy the offensive odour. Other subjects kept three days, at the same time, offered little trace of decomposition.

To give this strong drastic purgative to a patient in the state now described is unwarrantable; to permit the anasarca to go to this extent ere it is employed for its removal is unnecessary, because we have safer, and $I$ am sure much more efrectual, means. No advantage results by allowing the dropsy to accumulate, and much is lost if it only destroys the patient's confidence. There has been so close a connection between the exhibition of this drug and the patient's rapid declension, as shall terrify me from its use at any time, unless in the early stage of active anasarca, other medicines failing.

The only chance of obstructing the advance of the disease appears to be the removal of the causes that have induced it. The luxuries of the more affluent must be curtailed, and a return to more suitable and unstinulating diet and exercise enjoined. In reference to the less fortunate class, their laborivus exercises should be exchanged for lighter, and the drink should be restricted to a pint of porter or table-beer each day.

\section{Wymondham, Leicestershire, June 27, 1842 .}

P.S.-Since these remarks were penued, now nearly three months back, I have met with one or two cases in which the puncture by needles has been directed. A good quantity of fuid has been evacuated, and relief for the time afforded. 